\title{
Effective Viscoelastic Properties of One-Dimensional Composites
}

\author{
0.L. Cruz-González ${ }^{1}$, R. Rodríguez-Ramos ${ }^{2}$, J. Bravo-Castillero², R. Martínez-Rosado ${ }^{3}$ \\ R. Guinovart-Díaz ${ }^{2}$, J.A. Otero ${ }^{3}$, F. J. Sabina ${ }^{4}$
}

${ }^{1}$ Facultad de Ciencias Técnicas, Departamento de Matemática,Universidad de Matanzas, Varadero road

Km. 2 1/2, Matanzas, Cuba

2Facultad de Matemática y Computación, Universidad de La Habana, San Lázaro y L, Vedado

La Habana. CP 10400. Cuba

${ }^{3}$ Instituto Tecnológico de Estudios Superiores de Monterrey CEM, Atizapán de Zaragoza

EM CP 52926, México

${ }^{4}$ Instituto de Investigaciones en Matemáticas Aplicadas y en Sistemas, Universidad Nacional Autónoma de México, Apartado Postal 20-126, Delegación de Álvaro Obregón 01000 México, DF., México

Received Date: March 14, 2017 Accepted Date: May 18, 2017

Published Date: June 15, 2017

\begin{abstract}
In this paper, the use of Asymptotic Homogenization Method (AHM) is proposed to solve partial differential equations that describe the behavior of some viscoelastic heterogeneous materials. The mathematical statement of the problem is formulated. A theoretical and organized description of the AHM is exposed. Analytical expressions of the effective properties for heterogeneous viscoelastic materials, using the Laplace Transform and its inverse, are reported. Various viscoelastic kernels are considered, including Rabotnov's fractional-exponential kernel, to describe the behavior of laminate viscoelastic composites. Finally, numerical results are obtained to validate the use of the method.
\end{abstract}

Keywords: Asymptotic Homogenization Method - viscoelastic materials — effective coefficient - Laplace Transform - Rabotnov's kernel.

\section{INTRODUCTION}

Unidirectional fiber polymer composites show evidence of significant viscoelastic properties under shear and the last (ones) in turn induce viscoelastic properties of layered laminates. The most common approach to description of long term loading is in use of constitutive hereditary equations (see [1], [2], [7], [8] and [10]) or relationships following from them (see [5], [3] and [9]). Engineering practice is confined by creep, creep and recovery or constant rate tests of crossply composites to define viscoelastic properties.

It is of value to choose of suitable approximating function that is a kernel of hereditary equation. For the most part these are powerlaw (see [9], [11], [5] and [3]) or exponential creep (see[8], [9] and [10]) relations. To improve data approximation in [9] linear combination of powerlaw and exponential functions was used. Rabotnov's fractional-exponential function [1] possesses properties of power-law (shorter time) and exponential (longer time) functions. Nonlinear viscoelastic behavior is generally represented as the extension of linear models (see [1], [2], [7] and [10]), taking into account some irreversible effects. Of great importance is interrelation of creep and relaxation properties (see [1], [2], [6] and [7]). From analytical point of view, interrelation of viscoelastic properties implies inverse of the constitutive equations or establishing interrelation between creep and relaxation functions. The procedure of the constitutive equations inverse can be established by means of Laplace transform or resolvent operator. This study in terms of resolvent operator properties was intended to obtain an efficient approach of establishing interrelation between the constitutive equations describing the viscoelastic properties of layered laminates.

Today is extended increasingly the utilization of differential equations in the study and model of natural phenomena and technological processes, particularly through conservation laws, as a general principle for building models. The inability to obtain exact solutions in the equations that describe various phenomena of physics, biology, the economy and other sciences, has led to the need to search methods of approximate solution to their solutions. 
Perturbation theory is responsible of providing these methods of construction of approximate solutions for families of problems in differential equations, which have certain characteristics as very small parameters and rapidly oscillating parameters and which would generate a very high computational cost if they were resolved by computational methods. The methods can be appreciated in the theore-tical design of composite materials, looking for effective properties without their manufacture; this causes a considerable saving of time and materials. Homogenization methods are of the more used for its effectiveness. Such techniques have important applications in the analysis of physical and mechanical problems of compounds, or in dispersed porous media, among others.

In this work, the use of Asymptotic Homogenization Method in double scale is proposed for solving partial differential equations that describe the behavior of some viscoelastic heterogeneous materials.

\section{MATHEMATICAL MODEL}

A viscoelastic bar of length $L$ is considered, subjected to the action of a volumetric force $f(x)$. The operator $\widehat{\mathbf{r}}(\bullet)$ is a viscoelastic operator. The aim is to find the displacement function $u(x, t)$ solution of the following static problem,

$$
(\Theta)\left\{\begin{array}{l}
P u(x, t)=f(x) \\
u(x, t) \mid x=0=u^{0} ; \forall t \in R \\
u(x, t) \mid x=L=u^{L} ; \forall t \in R \\
u(x, t) \mid t=0=0 ; \forall x \in[0 ; L]
\end{array}\right.
$$

where the operators involved are defined as follow

$$
\begin{gathered}
P(\bullet):=\frac{\partial}{\partial x}\left(\hat{r}(x, t)\left(\frac{\partial(\bullet)}{\partial x}\right)\right) \\
\hat{r}(\mathrm{x}, \mathrm{t})(\bullet):=\int_{0}^{t} \mathbf{r}(x, t-\tau) \frac{\partial(\bullet)}{\partial \tau} d \tau
\end{gathered}
$$

The operator $\hat{\mathbf{r}}$ in terms of the displacement, is rewritten

$$
\hat{r}(\mathrm{x}, \mathrm{t})\left(\frac{\partial u(x, t)}{\partial x}\right):=\int_{0}^{t} \mathbf{r}(x, t-\tau) \frac{\partial}{\partial \tau}\left(\frac{\partial u(x, t)}{\partial x}\right) d \tau
$$

$\mathbf{r}(x, t)$ is called creep kernel or viscoelastic modulus and represents a property inherent to the material, which is obtained experimentally. In this case, we consider the kernel with the following properties.

\section{Additional properties of the kernel}

a. $\mathbf{r}(x+n \varepsilon, t)=\mathbf{r}(x, t)$ for $n=1,2, \ldots$, and $\varepsilon<<\mathrm{L}$.

(E-periodic) (see Fig. 1)

b. $\mathbf{r}\left(\frac{x}{\varepsilon}, t\right)$ is 1-periodic in $y$, where $y=\frac{x}{\varepsilon}$ is a fast variable that varies depending of the microstructure of the material.

c. $\mathbf{r}(y, t) \in C^{\infty}\left(R^{2}\right) \quad$.

d. $\exists \alpha, \beta, t_{0}$ such that $0<\alpha \leq \mathbf{r}\left(y, t_{0}\right) \leq \beta<\infty, \forall y \in R$.

e. $f(x) \in C^{\infty}([0, L])$. 
The creep kernel becomes a rapidly oscillating property when $\varepsilon \downarrow 0$ taking into account the condition (a). The magnitude $\varepsilon$ is called period of the structure, besides, this length is very small relative to the length of the bar. Under these conditions can be used the asymptotic homogenization method AHM (see [12]). The study of change of creep kernel only is discussed in the segment of length $\varepsilon$ (called periodic cell).

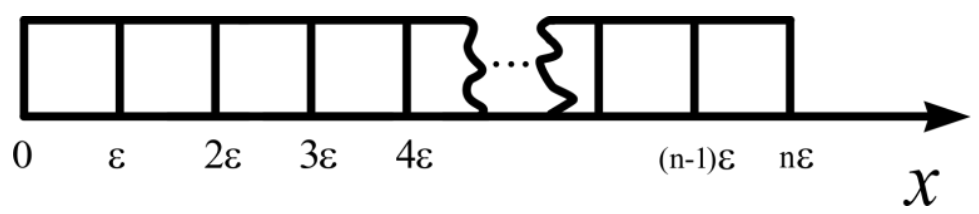

Fig1. Viscoelastic heterogeneous bar

The condition (b) is a consequence of introducing the variable $y=\frac{x}{\varepsilon}$ in the condition (a). This provides a second scale where is analyzed the fast or local variable $y$ whereas the variable $x$ is called slow or global variable.

\section{Solving $(\Theta)$ by two-scale homogenization method}

Homogenization techniques are used to obtain the effective behavior of the composite. Here, is proposed the two-scale homogenization technique to find the solution of the problem $(\Theta)$. The method consists in to build a formal asymptotic solution (see [12]) as solution of the problem. The asymptotic is defined as follows,

$$
\boldsymbol{u}(x, \varepsilon, t)=\sum_{i=0}^{\infty} \varepsilon^{i} u_{i}(x, y, t)
$$

where, $u_{i}(x, y, t)=u_{i}(x, y+1, t) \forall i, \forall x \in[0, L]$ and $\forall t \in R$ (means that, they are one periodic regarding to the variable y) and $u_{i} \in C^{\infty}\left([0, L] \times R^{2}\right), \forall i$.

If is applied the operator $\frac{\partial(\bullet)}{\partial x}$ to any function $u_{i}(x, y, t)$ in $(4)$, by the chain rule it is transformed

$$
\frac{\partial(\bullet)}{\partial x} \equiv \frac{\partial(\bullet)}{\partial x}+\frac{1}{\varepsilon} \frac{\partial(\bullet)}{\partial y}
$$

Let's define the following operator

$$
L_{\alpha \beta}(\bullet):=\frac{\partial}{\partial \alpha}\left(\hat{\mathbf{r}}(y, t)\left(\frac{\partial(\bullet)}{\partial \beta}\right)\right)
$$

for $\alpha, \beta=x, y$ indistinctly.

Taking the asymptotic (4) as the approximation of the exact solution of the problem $(\Theta)$, it is replaced into the left hand side of the differential equation, having in account (5) and (6). Equaling in powers of both members, are obtained the following problems,

$$
\begin{gathered}
\varepsilon^{-2}: L_{y y}\left(u_{0}\right)=0, \\
\varepsilon^{-1}: L_{x y}\left(u_{0}\right)+L_{y x}\left(u_{0}\right)+L_{y y}\left(u_{1}\right)=0, \\
\varepsilon^{0}: L_{x x}\left(u_{0}\right)+L_{x y}\left(u_{1}\right)+L_{y x}\left(u_{1}\right)+L_{y y}\left(u_{2}\right)-f(x)=0 .
\end{gathered}
$$

Besides, replacing (4) into the Dirichlet boundary conditions can be assumed,

$$
\begin{gathered}
u_{0}(0,0, t)=u^{(0)}, \quad u_{0}\left(L, \frac{L}{\varepsilon}, t\right)=u^{(L)}, \\
u_{i}(0,0, t)=0, \quad u_{i}\left(L, \frac{L}{\varepsilon}, t\right)=0, \forall i>0 .
\end{gathered}
$$


Results in (10) and (11) are justified by the fact that the formal asymptotic solution is obtained by construction; then is taken of this way for convenience.

In order to solve (7)-(8) can be used in each case a differential equation of the form $L_{y y}\left(u_{i}\right)=F$. The following lemma is needed. (see proof in [13])

Lemma 1: Consider the differential equation

$$
\frac{\partial}{\partial y_{i}}\left(K_{i j}^{\varepsilon} \frac{\partial \phi}{\partial y_{j}}\right)=F
$$

in a $Y$-cell, where $\phi$ is $Y$-periodic and $F \in L^{2}(Y)$. Then the following statements are satisfied

(i) Exists a solution Y-periodic $\phi$ if and only if $\langle F\rangle=0$.

(ii) If the solution Y-periodic $\phi$ exists, then it is unique up to an additive constant.

Is necessary to find the functions $u_{\mathrm{i}}$ with $i=0,1,2$ such that are satisfied the equations (7)-(9). As the viscoelastic operator $\hat{\mathbf{r}}(x, t)$ depends on the time variable, this makes that be possible to apply the Laplace Transform or the Laplace-Carson version and the result can be manipulated algebraically. From now on, the functions in Laplace's space will be written in capital letter and dependent of parameter $(p)$. The same notation, with the symbol $(\sim)$, denotes Laplace-Carson's space.

Problem for $\varepsilon^{-2}$

The problem (7) can be presented as follows

$$
\begin{aligned}
L_{y y} u_{0}(x, y, t) & =0, \\
\left.u_{0}(x, y, t)\right|_{x=0} & =u^{0}, \quad \forall t \in R \\
\left.u_{0}(x, y, t)\right|_{x=L} & =u^{L}, \quad \forall t \in R \\
\left.u_{0}(x, y, t)\right|_{t=0} & =0, \quad \forall x \in[0, L] .
\end{aligned}
$$

\section{Solution}

Substituting (6) into equation (12), it is obtained

$$
\begin{aligned}
& \frac{\partial}{\partial y}\left(\hat{\mathbf{r}}(y, t)\left(\frac{\partial u_{0}(x, y, t)}{\partial y}\right)\right)=0, \\
& \hat{\mathbf{r}}(y, t)\left(\frac{\partial u_{0}(x, y, t)}{\partial y}\right)=c(x, t),
\end{aligned}
$$

using (3),

$$
\int_{0}^{t} \hat{\mathbf{r}}(y, t-\tau) \frac{\partial}{\partial \tau}\left(\frac{\partial u_{0}(x, y, \tau)}{\partial y}\right) d \tau=c(x, t) .
$$


The following change of notation is taken

$$
\begin{gathered}
\frac{\partial u_{0}(x, y, t)}{\partial y}=w_{1}(x, y, t), \\
\int_{0}^{t} r(y, t-\tau) \frac{\partial}{\partial \tau}\left(w_{1}(x, y, t)\right) d \tau=c(x, t)
\end{gathered}
$$

Applying the Laplace-Carson transform to (14)

$$
L_{C}\left[\int_{0}^{t} \mathbf{r}(y, t-\tau) \frac{\partial}{\partial \tau}\left(w_{1}(x, y, \tau)\right) d \tau\right]=L_{C}[c(x, t)],
$$

and using the Convolution Theorem,

$$
L_{C}\left[\int_{0}^{t} g(t-\tau) \frac{f(\tau)}{d \tau} d \tau\right]=\widetilde{G}(p) *(\widetilde{F}(p)-f(0))
$$

is obtained

$$
\widetilde{R}(y, p) *\left(\widetilde{W}_{1}(x, y, p)-w_{1}(x, y, 0)\right)=\widetilde{C}(x, p),
$$

and from (12.3) and (13) is obtained $w_{1}(x, y, 0)=0$. Continuing with the analysis,

$$
\begin{gathered}
\widetilde{R}(y, p) * \widetilde{W}_{1}(x, y, p)=\tilde{C}(x, p), \\
\tilde{W}_{1}(x, y, p)=\tilde{C}(x, p) * \tilde{R}(y, p)^{-1} .
\end{gathered}
$$

The average over the cell, defined as

$$
\langle F(y)\rangle=\int_{0}^{1} F(y) d y,
$$

is applied to (16),

$$
\begin{aligned}
\left\langle\widetilde{W}_{1}(x, y, p)\right\rangle & =\left\langle\widetilde{C}(x, p) * \widetilde{R}(y, p)^{-1}\right\rangle, \\
\left\langle\widetilde{W}_{1}(x, y, p)\right\rangle & =\widetilde{C}(x, p) *\left\langle\widetilde{R}(y, p)^{-1}\right\rangle .
\end{aligned}
$$

As $u_{0}(x, y, t)$ is 1-periodic related to the variable $y$ then $\frac{\partial u_{0}(x, y, t)}{\partial y}$ is 1-periodic; property that is transmitted to $\widetilde{W}_{1}(x, y, p)$ because the transformed doesn't act on the variable $y$. Thus,

$$
\begin{gathered}
\left\langle\widetilde{W}_{1}(x, y, p)\right\rangle=0, \\
\widetilde{C}(x, p)=0 .
\end{gathered}
$$

Replacing (18) into (15)

$$
\widetilde{R}(y, p) * \widetilde{W}_{1}(x, y, p)=0,
$$

it is obtained

$$
\widetilde{W}_{1}(x, y, p)=0 .
$$


Applying the inverse Laplace-Carson Transform to both sides of (19),

$$
\begin{gathered}
L_{C}^{-1}\left[\widetilde{W}_{1}(x, y, p)\right]=L_{C}^{-1}[0], \\
\frac{\partial u_{0}(x, y, t)}{\partial y}=0 .
\end{gathered}
$$

Notice from (20) that this expression does not depend on $y$, then

$$
u_{0}(x, y, t)=v(x, t)
$$

Besides, from (12.1), (12.2) and (12.3) it follows that

$$
\begin{gathered}
u_{0}(0,0, t)=v(0, t)=u^{0}, \quad \forall t \in R \\
u_{0}\left(L, \frac{L}{\varepsilon}, t\right)=v(L, t)=u^{L}, \quad \forall t \in R \\
\left.u_{0}(x, y, t)\right|_{\mathrm{t}=0}=0, \forall x \in[0, L]
\end{gathered}
$$

From the expressions (8) and (21) can be considered that

$L_{x y}\left(u_{0}(x, y, t)\right)=L_{x y}(v(x, t))=0$. In this case the problem (8) is stated as follows

$$
\begin{array}{ll}
L_{y y}\left(u_{1}(x, y, t)\right) & =\quad-L_{y x}\left(u_{0}(x, y, t)\right), \\
\left.u_{1}(x, y, t)\right|_{x=0} & =0, \quad \forall t \in R \\
\left.u_{1}(x, y, t)\right|_{x=L} & =0, \quad \forall t \in R \\
\left.u_{1}(x, y, t)\right|_{t=0} & =0, \quad \forall x \in[0, L] .
\end{array}
$$

\section{Solution}

The problem in (22) has the form $L_{y y} u_{1}=F$ were $u_{1}$ is a 1-periodic function and $u_{0}$ is infinitely differentiable function, then the right hand side of (22) belongs to $L^{2}(R)$. The hypotheses of Lemma 1 are satisfied.

Therefore, if $\langle F\rangle=0$ is verified then the existence of a 1-periodic solution is guaranteed for the problem. Indeed

$$
\begin{gathered}
\langle F\rangle=\left\langle-L_{y x}(v(x, t))\right\rangle=-\left\langle\frac{\partial}{\partial y}\left(\hat{\mathbf{r}}(y, t)\left(\frac{\partial v(x, t)}{\partial x}\right)\right)\right\rangle \\
=-\left\langle\frac{\partial}{\partial y}(\hat{\mathbf{r}}(y, t))\left(\frac{\partial v(x, t)}{\partial x}\right)\right\rangle=-\left(\frac{\partial v(x, t)}{\partial x}\right)\left\langle\frac{\partial}{\partial y}(\hat{\mathbf{r}}(y, t))\right\rangle \\
=-\left(\frac{\partial v(x, t)}{\partial x}\right) \int_{0}^{1} \frac{\partial}{\partial y}(\hat{\mathbf{r}}(y, t)) d y=-\left.\left(\frac{\partial v(x, t)}{\partial x}\right) \hat{\mathbf{r}}(y, t)\right|_{0} ^{1}=0 .
\end{gathered}
$$

As creep kernel $\mathbf{r}(y, t)$ is 1-periodic then the difference between operators is canceled and it is obtained $\langle F\rangle=0$. 
The solution of the problem (22) is proposed as follows

$$
u_{1}(x, y, t)=m(y, t) \frac{\partial v(x, t)}{\partial x}+k(x, t),
$$

where $m(y, t)$ and $k(x, t)$ are infinitely differentiable functions and $m(y, t)$ is 1-periodic, besides it is assumed $m(0, t)=m(1, t)=0$.

This general form is obtained by separation of variables in the problem (22). Incorporating the condition (ii) of Lemma 1 and using the boundary conditions of the problem (22.1) and (22.2) it yields

$$
\begin{aligned}
& u_{1}(0,0, t)=m(y, t) \frac{\partial v(0, t)}{\partial x}+k(0, t)=k(0, t)=0, \\
& u_{1}\left(L, \frac{L}{\varepsilon}, t\right)=m\left(\frac{L}{\varepsilon}, t\right) \frac{\partial v(L, t)}{\partial x}+k(L, t)=k(L, t)=0 .
\end{aligned}
$$

Therefore, the function $k(x, t)$ can be zero for all $x \in[0, L]$ and $t \in R$.

Substituting equation (24) into (22) is obtained

$$
\begin{gathered}
L_{y y}\left(u_{1}(x, y, t)\right)=-L_{y x}(v(x, t)), \\
L_{y y}\left(m(y, t) \frac{\partial v(x, t)}{\partial x}\right)=-L_{y x}(v(x, t)),
\end{gathered}
$$

using (6) into (25) and solving it

$$
\begin{aligned}
& \left.\frac{\partial}{\partial y}\left(\hat{\mathbf{r}}(y, t)\left(\frac{\partial}{\partial y}\left(m(y, t) \frac{\partial v(x, t)}{\partial x}\right)\right)\right)\right\}=-\frac{\partial}{\partial y}\left(\hat{\mathbf{r}}(y, t)\left(\frac{\partial v(x, t)}{\partial x}\right)\right) \\
& \frac{\partial}{\partial y}\left(\hat{\mathbf{r}}(y, t)\left(\frac{\partial}{\partial y} m(y, t)+1\right)\right) \mid \frac{\partial v(x, t)}{\partial x}=0 .
\end{aligned}
$$

From (26) is obtained

$$
\begin{aligned}
& \frac{\partial}{\partial y} \hat{\mathbf{r}}(y, t)\left(\frac{\partial}{\partial y} m(y, t)+1\right)=0 \\
& \quad \frac{\partial}{\partial y}\left(\hat{\mathbf{r}}(y, t)\left(\frac{\partial}{\partial y} m(y, t)\right)\right)=-\frac{\partial}{\partial y}(\hat{\mathbf{r}}(y, t)) .
\end{aligned}
$$

Notice that (27) is of the form $L_{y y} m=F$ and as was discussed, from (23) is obtained $\left\langle\frac{\partial}{\partial y}(\hat{\mathbf{r}}(y, t))\right\rangle=0$. This makes that the hypothesis of Lemma 1 are satisfied. Therefore, the existence of a solution $m(y, t) 1$-periodic for (27) is insured.

To find the function $m(y, t)$ is necessary to solve the so-called cell or local problem,

\section{Local problem}

The local problem is stated as follows

$$
\frac{\partial}{\partial y}\left(\hat{\mathbf{r}}(y, t)\left(\frac{\partial}{\partial y} m(y, t)+1\right)\right)=0
$$




$$
\begin{aligned}
& m(0, t)=m(1, t)=0, \quad \forall t \in R \\
& m(y, 0)=-y, \quad \forall y \in\left[\begin{array}{ll}
0 & 1
\end{array}\right]
\end{aligned}
$$

The initial condition (28.2) is taken for convenience.

\section{Solution of the local problem}

Solving the Eq. (28) we have

$$
\hat{\mathbf{r}}(y, t)\left(\frac{\partial}{\partial y} m(y, t)+1\right)=c_{2}(t)
$$

applying the Laplace-Carson transform in both sides of (29)

$$
L_{C}\left\lceil\int_{0}^{t} \mathbf{r}(x, t-\tau) \frac{\partial}{\partial \tau}\left(\frac{\partial}{\partial y} m(y, \tau)+1\right) d(\tau)\right\rceil=L_{C}\left[c_{2}(t)\right],
$$

and using the Convolution Theorem in (30), the following expression is derived

$$
\left.\widetilde{R}(y, p) \mid L_{C}\left\lceil\frac{\partial}{\partial y} m(y, t)+1\right\rceil-\left(\frac{\partial}{\partial y} m(y, 0)+1\right)\right\rceil=\widetilde{C}_{2}(p) .
$$

Now, from the initial condition (28.2), is obtained

$$
\begin{gathered}
\widetilde{R}(y, p)\left\lfloor\frac{\partial}{\partial y} \widetilde{M}(y, p)+1\right\rceil=\widetilde{C}_{2}(p) \\
\left\langle\frac{\partial}{\partial y} \widetilde{M}(y, p)+1\right\rangle=\left\langle\frac{\widetilde{C}_{2}(p)}{\widetilde{R}(y, p)}\right\rangle \\
\left\langle\frac{\partial}{\partial x} \tilde{M}(y, p)\right\rangle+1=\tilde{C}_{2}(p)\left\langle\widetilde{R}^{-1}(y, p)\right\rangle
\end{gathered}
$$

As $m(y, t)$ is 1-periodic in relation to the variable $y$, then $\tilde{M}(y, p)$ is 1-periodic as well. It implies that,

$$
\left\langle\frac{\partial}{\partial y} \widetilde{M}(y, p)\right\rangle=0
$$

From (32), finally is obtained,

$$
\tilde{C}_{2}(p)=\left\langle\tilde{R}^{-1}(y, p)\right\rangle^{-1}
$$

Substituting (33) into (31) and after some algebraic manipulations,

$$
\tilde{M}(y, p)=\left\langle\widetilde{R}^{-1}(y, p)\right\rangle^{-1} \int_{0}^{y} \frac{1}{\tilde{R}(\gamma, p)} d \gamma-\gamma
$$

The function (34) is the analytical solution for the local problem. The expression (34) is called local function and is given in the Laplace's space. 
Problem for $\varepsilon^{0}$

According to (9), the problem is stated

$$
\begin{aligned}
& L_{y y}\left(u_{2}(x, y, t)\right)=-L_{x x}\left(u_{0}\right)-L_{x y}\left(u_{1}\right)-L_{y x}\left(u_{1}\right)+f(x), \\
& \left.u_{2}(x, y, t)\right|_{x=0} \quad=\quad 0, \quad \forall t \in R \\
& \left.u_{2}(x, y, t)\right|_{x=L} \quad=\quad 0, \quad \forall t \in R \\
& \left.u_{2}(x, y, t)\right|_{t=0} \quad=\quad 0, \quad \forall x \in[0, L] .
\end{aligned}
$$

The problem (35) is given of the form $L_{y y} u_{2}=F$ where $u_{2}$ is 1-periodic. The functions $\mathrm{v}(\mathrm{x}, \mathrm{t})$ and $\mathrm{m}(\mathrm{y}, \mathrm{t})$ are infinitely differentiable functions, implying $F \in L^{2}(R)$. The hypotheses of Lemma 1 are satisfied. If the condition $\langle F\rangle=0$ is verified then insure the existence of a solution for the problem (35) and is guarantee that the formal asymptotic solution can be constructed.

Indeed, this is possible

$$
\langle F\rangle=\left\langle-L_{x x}\left(u_{0}\right)-L_{x y}\left(u_{1}\right)-L_{y x}\left(u_{1}\right)+f(x)\right\rangle .
$$

Substituting (24) and (24) into (36),

$$
\begin{gathered}
\left\langle-L_{x x}(v(x, t))-L_{x y}\left(m(y, t) \frac{\partial v(x, t)}{\partial x}\right)-L_{y x}\left(m(y, t) \frac{\partial v(x, t)}{\partial x}\right)+f(x)\right\rangle \\
=-\left\langle L_{x x}(v(x, t))\right\rangle-\left\langle L_{x y}\left(m(y, t) \frac{\partial v(x, t)}{\partial x}\right)\right\rangle \\
-\left\langle L_{y x}\left(m(y, t) \frac{\partial v(x, t)}{\partial x}\right)\right\rangle+f(x) .
\end{gathered}
$$

From (37), is solved the expression denoted by (A),

$$
\left.\left\langle L_{y x}\left(m(y, t) \frac{\partial v(x, t)}{\partial x}\right)\right\rangle=\left\langle\frac{\partial}{\partial y}\left(\hat{\mathbf{r}}(y, t)\left(\frac{\partial}{\partial x}\left(m(y, t) \frac{\partial v(x, t)}{\partial x}\right)\right)\right)\right)\right\rangle
$$

Thus, using (6), the expression (37) is transformed

$$
\begin{gathered}
=-\left\langle\frac{\partial}{\partial x}\left(\hat{\mathbf{r}}(y, t)\left(\frac{\partial v(x, t)}{\partial x}\right)\right)\right\rangle-\left\langle\frac{\partial}{\partial x}\left(\hat{\mathbf{r}}(y, t)\left(\frac{\partial}{\partial y} m(y, t) \frac{\partial v(x, t)}{\partial x}\right)\right)\right\rangle+f(x), \\
=-\left\langle\hat{\mathbf{r}}(y, t)+\hat{\mathbf{r}}(y, t) \frac{\partial}{\partial y} m(y, t)\right\rangle \frac{\partial}{\partial x}\left(\frac{\partial v(x, t)}{\partial x}\right)+f(x),
\end{gathered}
$$




$$
=-\underbrace{\left\langle\hat{\mathbf{r}}(y, t)\left(\frac{\partial}{\partial y} m(y, t)+1\right)\right\rangle \frac{\partial}{\partial x}\left(\frac{\partial v(x, t)}{\partial x}\right)+f(x) .}_{(B)}
$$

Previously, in the local problem (see (29), (31) and (33)) was analyzed the term denoted by (B).

In view of the foregoing we obtain

$$
=-L_{C}^{-1}\left\lceil\left\langle\widetilde{R}^{-1}(y, p)\right\rangle^{-1}\right\rceil \frac{\partial}{\partial x}\left(\frac{\partial v(x, t)}{\partial x}\right)+f(x) .
$$

The requirement $\langle F\rangle=0$ is necessary, Therefore is obtained the homogenized problem

$$
L_{C}^{-1}\left[\left\langle\widetilde{R}^{-1}(y, p)\right\rangle^{-1}\right]\left(\frac{\partial^{2} v(x, t)}{\partial x^{2}}\right)=f(x)
$$

where the solution is denoted by $\mathrm{v}(\mathrm{x}, \mathrm{t})$. Besides, are taken the following Dirichlet boundary conditions,

$$
\begin{array}{ll}
v(0, t)=u^{0}, & \forall t \in R \\
v(L, t)=u^{L}, & \forall t \in R
\end{array}
$$

and the initial condition

$$
v(x, 0)=0, \quad \forall x \in[0, L] .
$$

The coefficient

$$
h(x, t)=L_{C}^{-1}\left[\left\langle\widetilde{R}^{-1}(x, p)\right\rangle^{-1}\right]
$$

is the homogenized or effective coefficient and will be denoted for the variable $h$.

\section{EFFECTIVE COEFFICIENTS FOR ONE-DIMENSIONAL TWO-PHASE VISCOELASTIC COMPOSITES}

Two-phase viscoelastic composites made of two different homogeneous materials are considered, in other words, $r^{(\delta)}(y, t) \equiv r^{(\delta)}(t) \quad$ with $\delta=1,2$. In the Fig 2 is shown the periodic cell of length $\varepsilon$, related to the local variable, where the supra-index $\delta=1,2$ indicates the corresponding material.

The creep kernel is taken as follows

$$
r^{(\delta)}=\left\{\begin{array}{lll}
r^{(1)}(t) & \text { if } & y \in \Omega^{(1)}, \\
r^{(2)}(t) & \text { if } & y \in \Omega^{(2)}
\end{array}\right.
$$

for a region $\Omega$ that is made of two regions, $\Omega^{(\delta)}, \delta=1,2$ where $\Omega^{(1)}=(0 \gamma]$ and $\Omega^{(2)}=(\gamma 1)$, it means that, $\Omega=[01]\{0\} \cup \Omega^{(1)} \cup \Omega^{(2)} \cup\{1\}$.

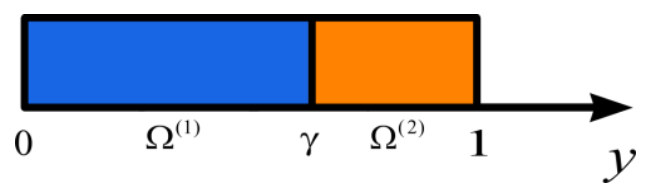

Fig2. Periodic cell for a two-phase one-dimensional composite

Taking the structure of the bar in account and based on that the creep kernels are homogeneous in $\Omega^{(\delta)}$ respectively, the effective coefficient (39) is transformed as follows 


$$
\begin{gathered}
\widetilde{H}(p)=\left\langle\widetilde{R}^{-1}(p)\right\rangle^{-1}=\frac{1}{\left\langle R^{-1}(p)\right\rangle}=\frac{1}{\int_{0}^{1} \widetilde{R}^{-1}(p) d y}=\frac{1}{\int_{0}^{1} \frac{1}{\widetilde{R}(p)} d y} \\
=\frac{1}{\gamma}=\frac{\widetilde{R}^{(1)}(p) \widetilde{R}^{(2)}(p)}{(1-\gamma) \tilde{R}^{(1)}(p)+\gamma \tilde{R}^{(2)}(p)} . \\
\int_{0}^{1} \frac{1}{\widetilde{R}^{(1)}(p)} d y+\int_{\gamma}^{1} \frac{1}{\widetilde{R}^{(2)}(p)} d y \quad \\
h(t)=L_{c}^{-1}[\widetilde{H}(p)]=L_{C}^{-1}\left\lfloor\frac{\tilde{R}^{(1)}(p) \tilde{R}^{(2)}(p)}{\left\lfloor(1-\gamma) \tilde{R}^{(1)}(p)+\gamma \tilde{R}^{(2)}(p)\right.}\right\rfloor
\end{gathered}
$$

In (40) is written the general form to compute the effective coefficient for two-phase one-dimensional viscoelastic composite.

\section{Example 1}

Now, we study a particular type of one-dimensional composite. A two-phase viscoelastic composite with the following creep kernels is considered

$$
r^{(\delta)}= \begin{cases}r^{(1)}(t)=a & \text { if } \quad y \in \Omega^{(1)}, \\ r^{(2)}(t)=b e^{-c t} & \text { if } \quad y \in \Omega^{(2)},\end{cases}
$$

where $a, b, c \in R$ (see [14]).

Applying the Laplace-Carson transform to the above creep kernels $r^{(\delta)}$ for $\delta=1,2$ the expressions for the kernels are written as

$$
\begin{gathered}
\widetilde{R}^{(1)}(p)=L_{C}\left[r^{(1)}(t)\right]=L_{C}[a]=a, \\
\widetilde{R}^{(2)}(p)=L_{C}\left[r^{(2)}(t)\right]=L_{C}\left[b e^{-c t}\right]=\frac{b p}{c+p} .
\end{gathered}
$$

Replacing (41) and (42) into (40) and grouping conveniently, is obtained a simplified expression,

$$
\widetilde{H}(p)=\frac{\alpha p}{\beta+p},
$$

where

$$
\alpha=\frac{a b}{a(1-\gamma)+b} \quad \text { and } \quad \beta=\frac{a c(1-\gamma)}{a(1-\gamma)+b}
$$

To determine the inverse Laplace-Carson transform of (4) the Cauchy residue theorem is used (see [14], [19]). The procedure is the following

$$
f(t)=L^{-1}[F(p)]=\sum_{k=1}^{n} \operatorname{Res}\left(e^{p t} F(p), s_{k}\right),
$$

where $s_{1}, s_{2} \ldots, s_{n}$ are the poles of $F(p)$ and if $s_{k}$ is a pole of order $m$ of $F(p)$ then

$$
\operatorname{Res}\left(F(p) e^{p t}, s_{k}\right)=\frac{1}{(m-1) !} \lim _{p \rightarrow s_{k}} \frac{d^{m-1}}{d p^{m-1}}\left(p-s_{k}\right)^{m} F(p) e^{p t}
$$


Using (45) to calculate (43) it yields

$$
\begin{array}{r}
h(t)=L_{C}^{-1}[\widetilde{H}(p)]=L^{-1}\left\lceil\frac{\widetilde{H}(p)}{p}\right\rfloor=L^{-1}\left\lceil\frac{\alpha}{\beta+p}\right\rceil, \\
\operatorname{Res}\left(\frac{\alpha}{\beta+p} e^{p t}, \beta\right)=\lim _{p \rightarrow-\beta}(p+\beta) \frac{\alpha}{\beta+p} e^{p t}=\alpha e^{-\beta t}
\end{array}
$$

The corresponding curves of the effective coefficient as a function of time are showed in Fig 3 for several values of the constants $a, b, c$ and $\gamma$. The method to obtain the analytical expressions of the inverse Laplace-Carson transform is reported in [14]

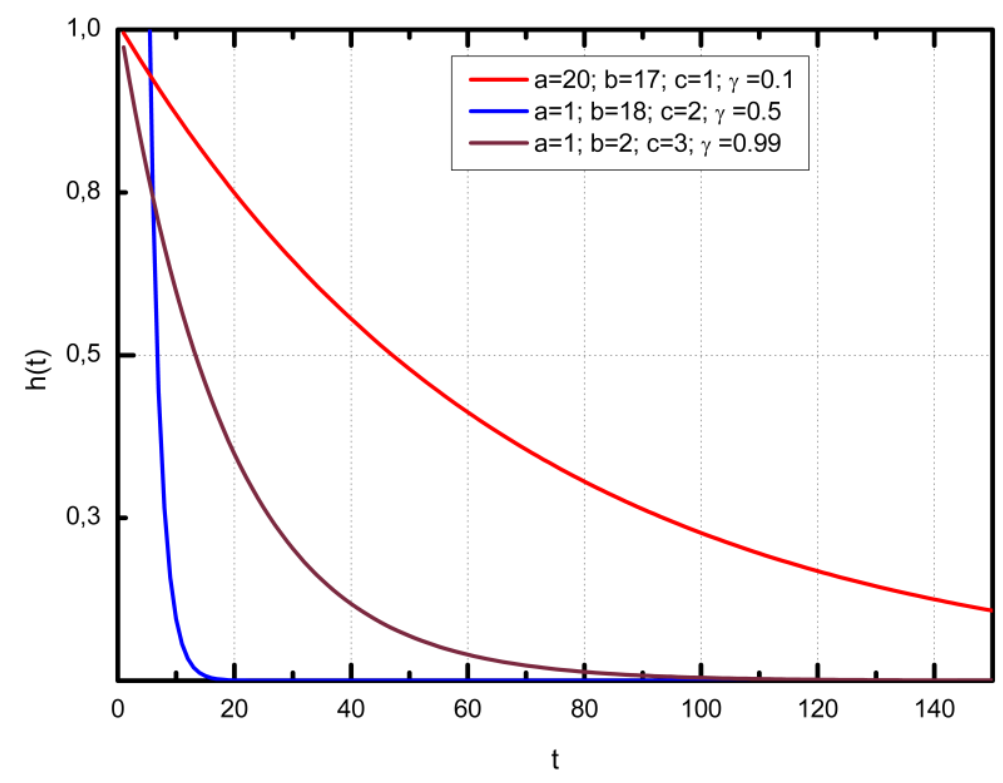

Fig3: Computation of the effective coefficient for different parameter values.

\section{Example 2}

The creep kernels that characterize the viscoelastic behavior of the material are taken as

$$
r^{(\delta)}=\left\{\begin{array}{l}
r^{(1)}(t)=a_{1}+b_{1} e^{-c_{1} t} \quad \text { if } \quad y \in \Omega^{(1)}, \\
r^{(2)}(t)=a_{2}+b_{2} e^{-c_{2} t} \quad \text { if } \quad y \in \Omega^{(2)}
\end{array}\right.
$$

where $a_{1}, a_{2}, b_{1}, b_{2}, c_{1}, c_{2} \in R \quad$ (see [14]).

Applying the Laplace-Carson transform to the above creep kernels $r^{(\delta)}$ for $\delta=1,2$

$$
\begin{gathered}
\tilde{R}^{(1)}(p)=L_{C}\left[r^{(1)}(t)\right]=L_{C}\left[a_{1}+b_{1} e^{-c_{1} t}\right]=a_{1}+\frac{b_{1} p}{p+c_{1}}, \\
\widetilde{R}^{(2)}(p)=L_{C}\left[r^{(2)}(t)\right]=L_{C}\left[a_{2}+b_{2} e^{-c_{2} t}\right]=a_{2}+\frac{b_{2} p}{p+c_{2}} .
\end{gathered}
$$

In the same way, substituting (48) and (49) into (40) and after some simplifications, the following expression is obtained (see [14]) 


$$
\widetilde{H}(p)=\frac{a_{N} p^{2}+b_{N} p+c_{N}}{a_{D} p^{2}+b_{D} p+c_{D}},
$$

where

$$
\begin{aligned}
a_{N} & =a_{1} a_{2}+a_{1} b_{2}+a_{2} b_{1}+b_{1} b_{2}, \\
b_{N} & =a_{1} a_{2} c_{2}+a_{1} a_{2} c_{1}+a_{1} b_{2} c_{1}+a_{2} b_{1} c_{2}, \\
c_{N} & =a_{1} a_{2} c_{1} c_{2}, \\
a_{D} & =a_{1}+b_{1}-\gamma a_{1}-\gamma b_{1}+\gamma a_{2}+\gamma b_{2}, \\
b_{D} & =a_{1} c_{2}+a_{1} c_{1}+b_{1} c_{2}-\gamma a_{1} c_{2}-\gamma a_{1} c_{1}-\gamma b_{1} c_{2} \\
& +\gamma a_{2} c_{2}+\gamma a_{2} c_{1}+\gamma b_{2} c_{1}, \\
c_{D}= & a_{1} c_{1} c_{2}-a_{1} c_{1} c_{2} \gamma+a_{2} c_{1} c_{2} \gamma .
\end{aligned}
$$

As in the previous Example 1, (45) is used to calculate the inverse Laplace-Carson transform of (50)

$$
\begin{gathered}
h(t)=L_{C}^{-1}[\widetilde{H}(p)]=L^{-1}\left\lfloor\frac{\widetilde{H}(p)}{p}\right\rfloor \\
=L^{-1}\left\lfloor\frac{a_{N} p^{2}+b_{N} p+c_{N}}{p\left(a_{D} p^{2}+b_{D} p+c_{D}\right)}\right\rfloor=L^{-1}\left\lfloor\frac{A(p)}{p\left(p-s_{2}\right)\left(p-s_{3}\right)}\right\rfloor,
\end{gathered}
$$

where,

$$
A(p)=a_{N} p^{2}+b_{N} p+c_{N}
$$

and

$$
s_{1}=0, s_{2}=\frac{-b_{D}+\sqrt{b_{D}^{2}-4 a_{D} c_{D}}}{2 a_{D}}, s_{3}=\frac{-b_{D}-\sqrt{b_{D}^{2}-4 a_{D} c_{D}}}{2 a_{D}} .
$$

are the poles of $\frac{\widetilde{H}(p)}{p}$. Having into account (45) and (46),

$$
L^{-1}\left\lfloor\frac{A(p)}{p\left(p-s_{2}\right)\left(p-s_{3}\right)}\right\rceil=\sum_{k=1}^{3} \operatorname{Res}\left\lceil\frac{A(p) e^{p t}}{p\left(p-s_{2}\right)\left(p-s_{3}\right)}, s_{k}\right\rfloor .
$$

Finally

$$
h(t)=\frac{A(0)}{s_{2} s_{3}}+\frac{A\left(s_{2}\right) e^{s_{2} t}}{s_{2}\left(s_{2}-s_{3}\right)}+\frac{A\left(s_{3}\right) e^{s_{3} t}}{s_{3}\left(s_{3}-s_{2}\right)} .
$$

In (52) is obtained an analytical unified formula for the effective coefficient. For this purpose, it supposed that the order of the poles, once calculated, is always 1. If to some set of data, any one of the poles has major order than 1, then would have to do changes in (52) (see (45) and (46)). 
In the Fig4 is showed to calculate the effective coeficient a comparison between the use of the analytical expression (52) and the use of a numerical algorithm developed in MATLAB.

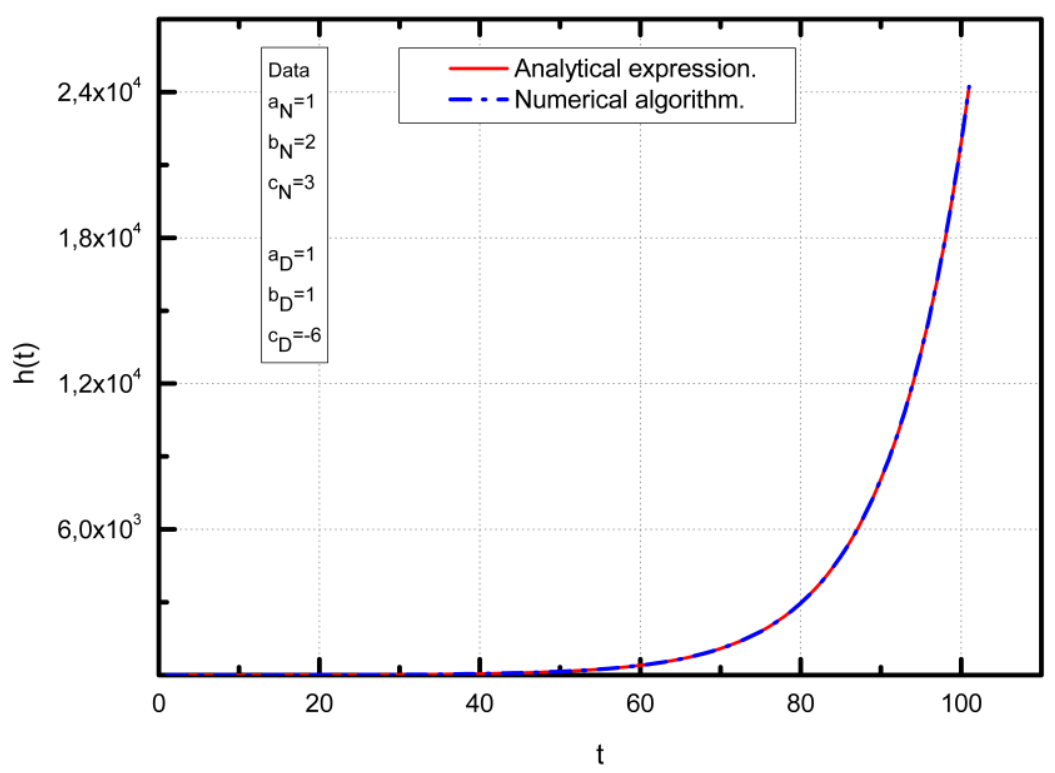

Fig4. Comparison between the use of the analytical expression (52) and the use of a numerical algorithm developed in MATLAB.

\section{Example 3}

In the two previous examples the proposed creep kernels were exponential functions. This facilitated the obtaining of analytical expressions of the effective coefficient. However, the exponential function kernels or their linear combinations, do not always describe correctly the viscoelastic behavior for some determined materials (see [17], [18]). The situation is solved with the use of fraction-exponential functions (see [21], [22])

$$
\ni_{\alpha}(\beta, t-\tau)=(t-\tau)^{\alpha} \sum_{n=0}^{\infty} \frac{(-\beta)^{n}(t-\tau)^{n(1+\alpha)}}{\Gamma[(n+1)(1+\alpha)]}
$$

with $-1<\alpha \leq 0$, as kernel of the viscoelastic operators. These enable an analytical obtaining of the results under the use of the Laplace transform and at the same time, present an excellent concordance with the experimental data (see[17], [18]). Taking the creep kernel as a fraction-exponential function or Rabotnov's kernel,

$$
r(t)=\lambda \mu_{0} \ni_{\alpha}(\beta, t)
$$

where

$$
\beta=\frac{1}{\tau^{1-\alpha}}, \lambda=\beta\left(1-\varepsilon_{\text {max }}\right)=\frac{\mu_{\infty}-\mu_{0}}{\mu_{0}} \beta
$$

$\tau$ is relaxation time, $\mu_{\infty}$ is the shear modulus at $t \rightarrow \infty, \mu_{0}$ is instantaneous shear modulus and $\varepsilon_{\max }$ is maximal shear strain. Then the viscoelastic behavior of the materials is described for the following four parameters $\mu_{0,}, \beta$ (or $\left.\tau\right)$ and $\lambda$ (see [17], [18]).

The algebraic properties of the Rabotnov's kernel are very well developed in [20]. One of the most important aspects that is exposed in the theory of these functions is the analytical way of its Laplace transform

$$
L\left[\ni_{\alpha}(\beta, t)\right] \equiv \int_{0}^{\infty} \ni_{\alpha}(\beta, t) e^{-p t}=\frac{1}{p^{1-\alpha}+\beta} .
$$


Applying the Laplace-Carson transform to the Rabotnov's kernels in (54) and according to (56) is obtained

$$
\begin{aligned}
& \tilde{R}^{(1)}(p)=L_{C}\left[r^{(1)}(t)\right]=p L\left[r^{(1)}(t)\right]=p L\left[\lambda_{1} \mu_{01} \ni_{\alpha}\left(\beta_{1}, t\right)\right]=\frac{\lambda_{1} \mu_{01} p}{x+\beta_{1}}, \\
& \tilde{R}^{(2)}(p)=L_{C}\left[r^{(2)}(t)\right]=p L\left[r^{(2)}(t)\right]=p L\left[\lambda_{2} \mu_{02} \ni_{\alpha}\left(\beta_{2}, t\right)\right]=\frac{\lambda_{2} \mu_{02} p}{x+\beta_{2}},
\end{aligned}
$$

where is taken the variable $x \equiv p^{1-\alpha}$.

Replacing (57) and (58) into (40) and after some simplifications, it is found an expression of the form

$$
\widetilde{H}(p)=\frac{M p}{x+x_{0}},
$$

where

$$
M=\frac{\lambda_{1} \mu_{01} \lambda_{2} \mu_{02}}{(1-\gamma) \lambda_{1} \mu_{01}+\gamma \lambda_{2} \mu_{02}}, \quad x_{0}=\frac{(1-\gamma) \lambda_{1} \mu_{01} \beta_{2}+\gamma \lambda_{2} \mu_{02} \beta_{1}}{(1-\gamma) \lambda_{1} \mu_{01}+\gamma \lambda_{2} \mu_{02}}
$$

To determine the inverse Laplace-Carson transform of (59) can be used (56)

$$
\begin{gathered}
h(t)=L_{C}^{-1}[\widetilde{H}(p)]=L^{-1}\left[\frac{H(p)}{p}\right] \\
=L^{-1}\left\lfloor\frac{M}{x+x_{0}}\right\rfloor=L^{-1}\left[\frac{M}{p^{1-\alpha}+x_{0}}\right]=M \ni_{\alpha}\left(x_{0}, t\right) .
\end{gathered}
$$

Finally, using the Rabotnov's kernel as the creep kernel to describe the behavior of the materials, is obtained in (61) an analytical expression to calculate the effective coefficient of the material. The great advantage that has the use of these fraction-exponential functions of Rabotnov because in addition to be a guarantee for the experimental data, they enable an elegant analytical modeling of the problem.

In the Fig 5 is showed the effective coefficient calculated in (61) for real values of the parameters $\mu_{0}, \alpha, \beta$ and $\lambda$ (taken from [18]) and its comparison with the numerical result.

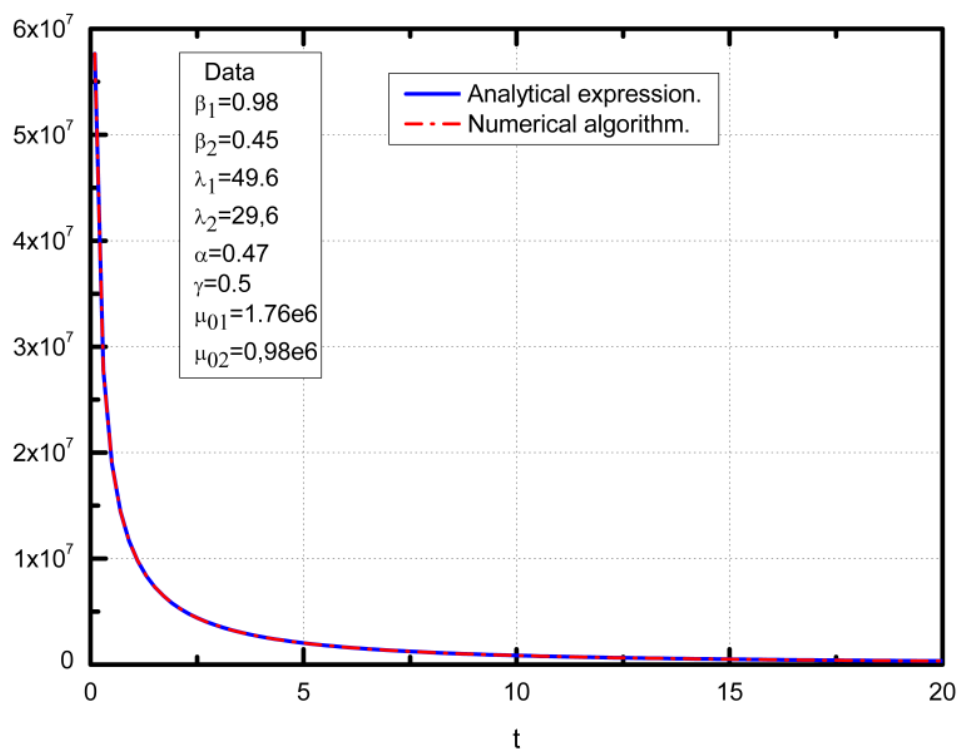

Fig5. The graphic of the effective coefficient 


\section{CONCLUSION}

The present work studies the behavior of one dimensional viscoelastic composites under different kernel functions. Using the two scale asymptotic homogenization technique, the local problems and effective coefficients are derived. A mathematical justification is given and shows the effectiveness of $A H M$ to solve one dimensional viscoelastic heterogeneous problems. Several analytical methods to calculate the Laplace transform are exposed. The use of fractional exponential functions reveals a great benefit for the computation of the effective properties. Besides, numerical results are shown using real parameters taken from the literature.

\section{REFERENCES}

1. Rabotnov, Y.N. (1977). Elements of hereditary mechanics of solids. Nauka. (in Russian)

2. Findley, W. N., Lai, J.S. \& Onaran K, K. (1976). Creep and relaxation of nonlinear viscoelastic materials. Inc. -NY: Dover Publications.

3. Brinson, Y.F. \& Dillard, D.A. (1982). The prediction of long term viscoelastic properties of fiber reinforced plastics. In ICCM-IV Progress in Sci \& Engng of Composites (pp. 787-793). Tokyo.

4. Zinoviev, P. \& Tairova, L. (1995). Identifying the Properties of Individual Plies Constituting Hybrid Composites. In Inverse Problems in Engineering, V2 (pp. 141-154).

5. Charentenay, F.X. \& Zaidi, M.A. (1982). Creep behavior of carbon-epoxy ( \pm 45$) 2$ s laminates. In ICCM-IV Progress in Sci \& Engng of Composites ( pp. 787-793). Tokyo.

6. Dumansky, A.M. \& Strekalov, V.B. (1999). Creep and relaxation of heritable orthotropic continua under the plane stress state. In Journ Strain Analysis for Engineering Design, V34 (pp. 361-367).

7. Oza, A., Vanderby, R. J. \& Lakes, R. (2003). Interrelation of creep and relaxation for nonlinearly viscoelastic materials: application to ligament and metal. In Rheol Acta, V42 (pp. 557-568).

8. Noh, J. \& Whitcomb , J. (2003). Efficient techniques for predicting viscoelastic behavior of sublaminates. In Composites: Part B: Engineering, V34 (pp. 727-736).

9. Deng, S., LI, X. \& Weitsmam, Y.J. (2003). Time-dependent Deformation of Stiched T300 Mat/Urethane 420 IMR Cross-ply Composite Laminates. In Mechanics of Time-Dependent Materials , V7 (pp. 41-69).

10. Miranda, R. \& Marques, A.T. (1998). Analytical and Experimental Evaluation of Nonlinear Viscoelastic-Viscoplastic Composite Laminates under Creep, Creep-Recovery, Relaxation and Ramp Loading. In Mechanics of Time-Dependent Materials, V2 (pp. 113-128).

11. Pang, F. \& Wang, C.H. (1999) Activation theory for creep of woven composites. In Composites: Part B, V30 (pp. 613-620).

12. Bakhvalov N., Panasenko G. (1989). Homogenisation: Averaging Processes in Periodic Media. London: Kluwer Academic.

13. Persson, L. E., Persson, L., Svanstedt, N. \& Wyller, J. (1993). The Homogenization Method. An Introduction. Student litteratur.

14. Liu, S., Chen, K-Z, \& Feng, X-A.(2004). Prediction of viscoelastic property of layered materials. International Journal of Solids and Structures 41, 3675-3688.

15. Craig, I. J. D., Thompson, A. M. \& Thompson, W. J. (1994). Practical Numerical Algorithms Why Laplace Transforms Are Difficult To Invert Numerically. Computers in Physics 8, 648; doi: 10.1063/1.4823347.

16. Maghous, S. \& Creus, G.J. (2003) Periodic homogenization in thermoviscoelasticity: case of multilayered media with ageing. International Journal of Solids and Structures 40, 851-870.

17. Sevostianov, I., Levin, V. \& Radi, E. (2016). Effective viscoelastic properties of short-fiber reinforced composites. International Journal of Engineering Science 100, 61-73.

18. Sevostianov, I. \& Levin, V. (n.d.) Creep and relaxation contribution tensors for spheroidal pores in hereditary solids: fraction-exponential operators approach. Is received by courtesy of the author. 
19. Debnath, L. (1995). Integral Transformation and Their Applications. New York: CRC Press.

20. Rabotnov, Yu.N. (1948). Equilibrium of an elastic medium with after-effects. J. Appl. Math. Mech. (PMM) 12, 53-62.

21. ScottBlair, G.W. \& Coppen,F.M.V.(1939). The subjective judgement of the elastic and plastic properties of soft bodies: the differential thres holds for viscosities and compression moduli. Proceedings of the Royal Society $B, 128,109-125$.

22. ScottBlair, G.W. \& Coppen, F.M.V. (1943). The estimation of firmness in soft materials. American Journal of Psychology, 56, 234-246.

Citation: O.L. Cruz-González, R. Rodríguez-Ramos, J. Bravo-Castillero, R.Martínez-Rosado, R. Guinovart-Díaz, J.A. Otero, F. J. Sabina. "Effective viscoelastic properties of one-dimensional composites", American Research Physics, vol 3, no. 1, 2017, pp. 1-17.

Copyright (C) 2017 O.L. Cruz-González, R. Rodríguez-Ramos, J. Bravo-Castillero, R.Martínez-Rosado, R. Guinovart-Díaz, J.A. Otero, F. J. Sabina, This is an open access article distributed under the Creative Commons Attribution License, which permits unrestricted use, distribution, and reproduction in any medium, provided the original work is properly cited. 